\title{
Formal Verification of Spacecraft Control Programs (Experience Report)
}

\author{
Andrey Mokhov \\ Newcastle University \\ United Kingdom \\ andrey.mokhov@ncl.ac.uk
}

\author{
Georgy Lukyanov \\ Newcastle University \\ United Kingdom \\ g.lukyanov2@ncl.ac.uk
}

\author{
Jakob Lechner \\ RUAG Space GmbH \\ Austria \\ jakob.lechner@gmx.net
}

\begin{abstract}
Verification of correctness of control programs is an essential task in the development of space electronics; it is difficult and typically outweighs design and programming tasks in terms of development hours. This experience report presents a verification approach designed to help spacecraft engineers reduce the effort required for formal verification of low-level control programs executed on custom hardware.

The verification approach is demonstrated on an industrial case study. We present REDFIN, a processing core used in space missions, and its formal semantics expressed using the proposed metalanguage for state transformers, followed by examples of verification of simple control programs.
\end{abstract}

\section{CCS Concepts - Software and its engineering;}

Keywords formal verification, instruction set architecture

ACM Reference Format:

Andrey Mokhov, Georgy Lukyanov, and Jakob Lechner. 2019. Formal Verification of Spacecraft Control Programs (Experience Report). In Proceedings of the 12th ACM SIGPLAN International Haskell Symposium (Haskell '19), August 22-23, 2019, Berlin, Germany. ACM, New York, NY, USA, 7 pages. https://doi.org/10.1145/3331545.3342593

\section{Introduction}

Software bugs play a major role in the history of spacecraft accidents [Leveson 2004]. Some of the known missionending bugs, e.g. due to software updates, would have been difficult to prevent, but integer overflows [Ben-Ari 2001] and incorrect unit conversion [NASA 1999] should have been eradicated long ago. This paper combines known formal verification and programming languages techniques and presents a formal verification approach for simple control tasks, such as satellite power management, which are executed on a real processing core used in space missions.

Fig. 1 shows an overview of our approach. The bottom part corresponds to conventional code generation and test,

Permission to make digital or hard copies of part or all of this work for personal or classroom use is granted without fee provided that copies are not made or distributed for profit or commercial advantage and that copies bear this notice and the full citation on the first page. Copyrights for thirdparty components of this work must be honored. For all other uses, contact the owner/author(s).

Haskell '19, August 22-23, 2019, Berlin, Germany

() 2019 Copyright held by the owner/author(s).

ACM ISBN 978-1-4503-6813-1/19/08.

https://doi.org/10.1145/3331545.3342593

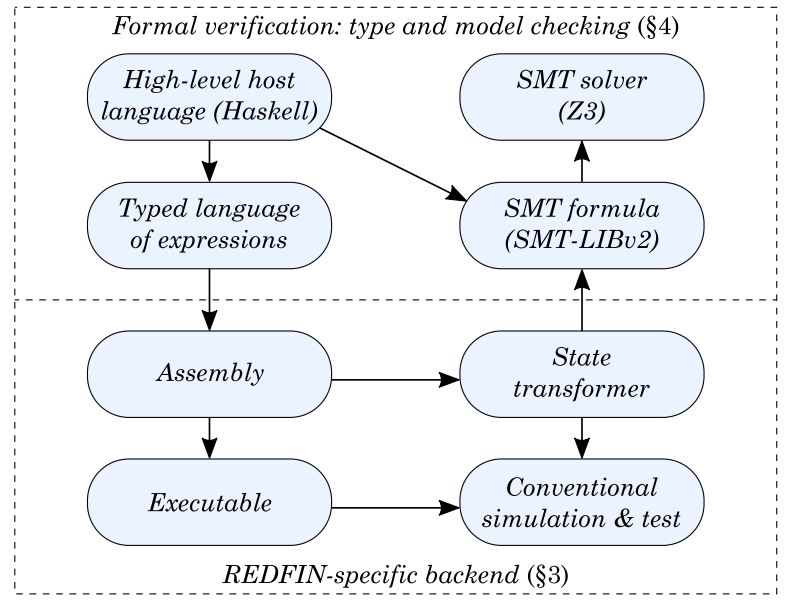

Figure 1. Overview of the presented verification approach.

where REDFIN ${ }^{1}$ assembly language is executed by simulating the effect of each instruction on the state of the processor and memory. The corresponding state transformer is typically implicit and intertwined with the rest of the simulation infrastructure. The main idea of our approach is to represent the state transformer explicitly so that it can be symbolically manipulated and used not only for simulation but also for formal verification. The latter is achieved by compiling state transformers to SMT formulas and using an SMT solver, e.g. Z3 [De Moura and Bjørner 2008], to verify that certain correctness properties hold, for example, that integer overflow cannot occur regardless of input parameters and that the program always terminates within stated time.

By using Haskell as the host language we can readily implement compilers from higher-level typed languages to untyped assembly, eradicating incorrect number and unit conversion bugs. As shown at the top of Fig. 1, engineers can write high-level control programs for the REDFIN architecture directly in a small subset of Haskell. These high-level programs can be used for type-safe code generation and as executable specifications of intended functionality for the purposes of program synthesis and equivalence checking.

We first introduce the REDFIN processing core ( 2 ), then present our verification approach (\$3-§4), and conclude by a discussion (§5) and a review of related work (§6).

\footnotetext{
${ }^{1}$ REDFIN stands for 'REDuced instruction set for Fixed-point \& INteger arithmetic'. This instruction set and the corresponding processing core were
} developed by RUAG Space Austria GmbH for space missions (see §2). 


\section{The REDFIN Architecture Overview}

Many spacecraft subsystems rely on integrated circuits to perform control tasks or simple data processing. Typically, these integrated circuits are realised with Field Programmable Gate Arrays (FPGAs) benefiting from their flexibility and low cost. Modern space-qualified FPGAs that can withstand radiation in Earth orbit or deep space have a limited amount of programmable resources, and it is often not feasible to implement a fully-fledged processor system in such an FPGA next to the mission-specific circuitry. The REDFIN instruction set was developed to address this issue and meet the following goals: (i) simple instruction set with a small hardware footprint, (ii) reduced complexity to support formal verification of programs, and (iii) deterministic real-time behaviour.

\subsection{REDFIN Instruction Set and Microarchitecture}

REDFIN instructions have a fixed width of 16 bits. The instruction set is based on a register-memory architecture, i.e. instructions can fetch their operands from registers as well as directly from the memory. This architecture favours a small register set, which minimises the hardware footprint of the processing core. Furthermore, the number of instructions in a program is typically smaller in comparison to traditional load/store architectures where all operands have to be transferred to registers before any operations can be performed. There are 47 instructions of the following types:

- Load/store instructions for moving data between registers and memory, and loading of immediate values.

- Integer and fixed-point arithmetic operations.

- Bitwise logical and shift operations.

- Control flow instructions and comparison operations.

- Bus access instructions for read \& write operations on an AMBA AHB bus (not covered in this paper).

The REDFIN processing core fetches instruction and data words from a small and fast on-chip SRAM. This only allows for execution of simple programs, however, it also eliminates the need to implement caches and thus removes a source of non-determinism of conventional processors. High performance is not one of the main goals, hence the core is not pipelined and does not need to resolve data/control hazards or perform any form of speculative execution. These properties greatly simplify worst-case execution time analysis.

\subsection{Requirements for Formal Verification}

Verification of functional correctness of REDFIN programs, as defined by a requirement specification, clearly is an essential task for the development of space electronics. There are also important non-functional requirements, such as worst-case execution time and energy consumption, which rely on the implementation guarantees provided by the processing core.

To reduce verification complexity, the REDFIN core only allows to execute a single subroutine whose execution is triggered by a higher-level controller in the system. The implementation guarantees that concurrent bus accesses to

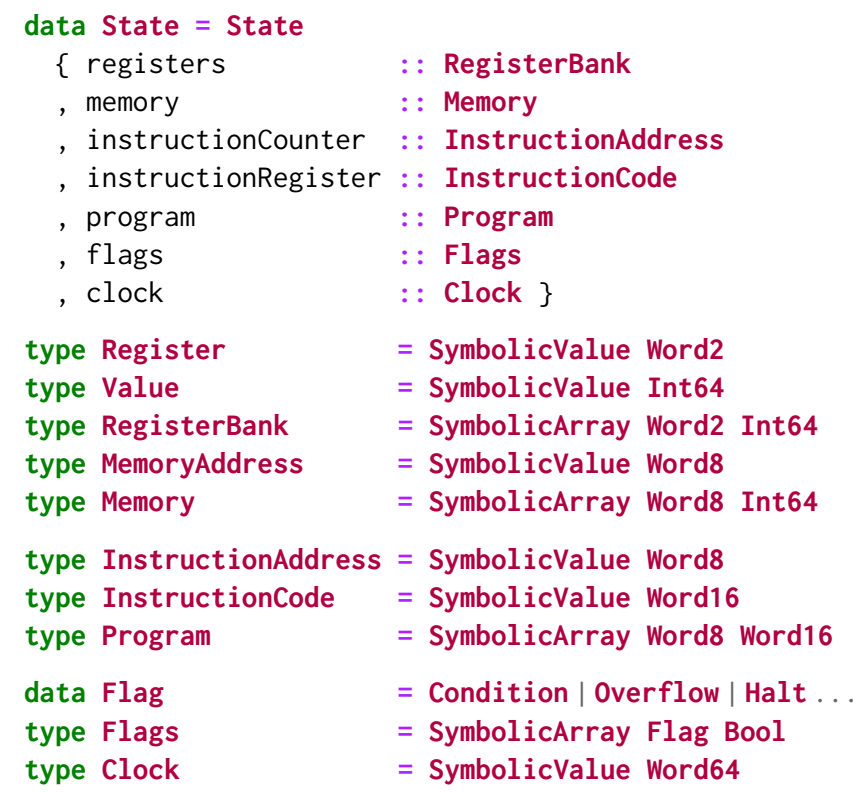

Figure 2. Basic types for modelling REDFIN.

the processor registers or memory do not affect the subroutine execution time. Furthermore, the processor does not implement interrupt handling. All these measures are taken to provide real-time subroutine execution guarantees and make the verification of non-functional properties feasible.

Despite these restrictions, the REDFIN core has already proven its effectiveness for simple control tasks and arithmetic computations as part of an antenna pointing unit for satellites. Nevertheless, verification can be difficult and timeconsuming, even for small and simple programs. Verification activities, following engineering standards for space electronics, typically outweigh programming and design tasks by a factor of two in terms of development hours. Usually verification is performed via program execution on an instruction set simulator or a hardware model of the processor. Manually deriving test cases from the specification is cumbersome and error-prone and simulation times can become prohibitively long with a large number of tests that are often needed to reach the desired functional and code coverage. Formal verification methods can prove that a program satisfies certain properties for all possible test cases and are therefore immensely valuable for completing the verification with superior efficiency and quality.

\section{Modelling REDFIN in Haskell}

In this section we formally define the REDFIN microarchitecture and express the semantics of the instruction set as an explicit and symbolic state transformer.

\subsection{The REDFIN Microarchitecture State}

The main idea of our approach is to use an explicit state transformer semantics of the REDFIN microarchitecture. The State of the entire processing core is a product of states of every component, see Fig. 2. We define SymbolicValue and 
SymbolicArray on top of the SBV library [Erkok 2019] that we use as a frontend for SMT translation and verification.

There are 4 registers (addressed by Word2) and 256 memory cells (addressed by Word8) that store 64-bit values (Int64). The register bank and memory are represented by symbolic arrays that can be accessed via SBV's functions readArray and writeArray. REDFIN uses 16-bit InstructionCodes, whose 6 leading bits contain the opcode, and the remaining 10 bits hold instruction arguments. The Program maps 8-bit instruction addresses to instruction codes.

The microarchitecture status Flags support conditional branching, track integer overflow, and terminate the program (we omit a few other flags for brevity). The Clock is a 64-bit counter incremented on each clock cycle. Status flags and the clock are used for diagnostics, formal verification, and worst-case execution time analysis.

\subsection{Instruction and Program Semantics}

We can now define the formal semantics of REDFIN instructions and programs as a state transformer $T: S \rightarrow S$, i.e. a function that maps states to states. We distinguish instructions and programs by using Haskell's list notation, e.g. $T_{\text {nop }}$ is the semantics of the instruction nop $\in I$, whereas $T_{[\text {nop] }}$ is the semantics of the single-instruction program $[$ nop] $\in P$.

Definition (program semantics): The semantics of a program $p \in P$ is inductively defined as follows:

The semantics of the empty program []$\in P$ coincides with the semantics of the instruction nop and is the identity state transformer: $T_{[]}=T_{\text {nop }}=$ id.

The semantics of a single-instruction program $[i] \in P$ is a composition of (i) fetching the instruction from the program memory $T_{\text {fetch }}$, (ii) incrementing the instruction counter $T_{\text {inc }}$, and (iii) the state transformer of the instruction itself $T_{i}$; or, using the order of state components from Fig. 2:

$$
\begin{aligned}
& T_{\text {fetch }}=(r, m, i c, i r, p, f, c) \mapsto(r, m, i c, p[i c], p, f, c+1) \\
& T_{\text {inc }}=(r, m, i c, i r, p, f, c) \mapsto(r, m, i c+1, i r, p, f, c) \\
& T_{[i]}=T_{\mathrm{i}} \circ T_{i n c} \circ T_{\text {fetch }}
\end{aligned}
$$

The semantics of a composite program $\mathrm{i}: \mathrm{p} \in P$, where the operator : prepends an instruction $i \in I$ to a program $\mathrm{p} \in P$, is defined as $T_{i: p}=T_{p} \circ T_{[i]}$.

We represent state transformers in Haskell using the state monad, a classic approach to emulating mutable state in a purely functional programming language [Wadler 1990]. We call our state monad Redfin and define it as follows:

$$
\begin{aligned}
& \text { data Redfin } a=\text { Redfin } \\
& \quad\{\text { transform : : State }->(a, \text { State) }\}
\end{aligned}
$$

A computation of type Redfin a yields a value of type a and possibly alters the State of the REDFIN microarchitecture. The type Redfin () describes a computation that does not produce any value as part of the state transformation; such computations directly correspond to state transformers.
For example, here is the state transformer $T_{\text {inc }}$ :

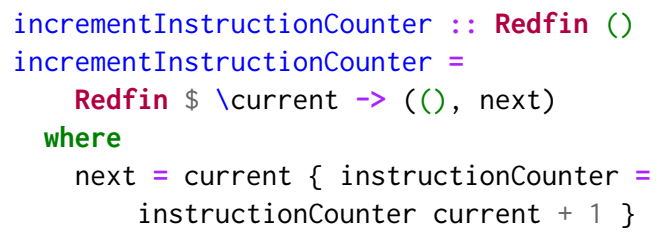

In words, the state transformer looks up the value of the instructionCounter in the current state and replaces it in the next state with the incremented value. We can compose such primitive computations into more complex state transformers using Haskell's do-notation:

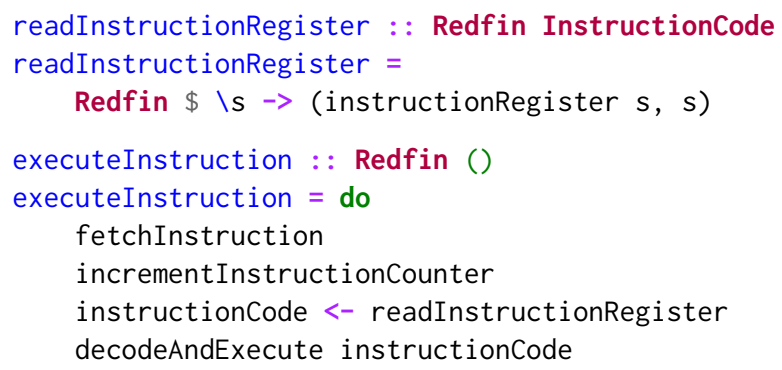

Here readInstructionRegister reads the instruction code from the current state without modifying it, and is subsequently used in executeInstruction, which defines the semantics of the REDFIN execution cycle. We omit definitions of fetchInstruction and decodeAndExecute for brevity. The latter is a case analysis of 47 opcodes that returns the matching instruction. We discuss several instructions below.

\subsubsection{Halting the Processor}

The instruction halt sets the flag Halt, thereby stopping the execution of the current subroutine until a new one is started by a higher-level system controller that resets Halt.

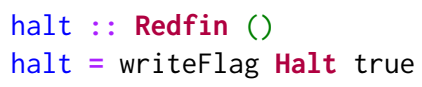

The auxiliary function writeFlag modifies the flag:

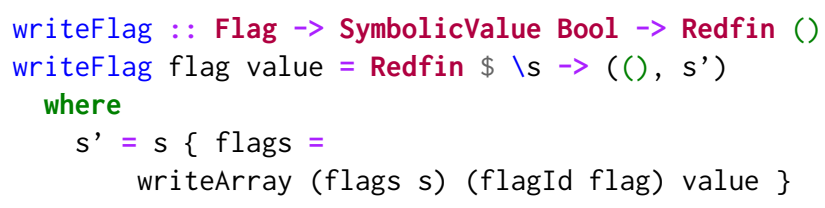

In the rest of the paper we will use auxiliary functions readRegister, writeRegister, readState, etc.; they are simple state transformers defined similarly to writeFlag.

\subsubsection{Arithmetics}

The instruction abs is more involved: it reads a register and writes back the absolute value of its contents. The semantics accounts for the potential integer overflow that leads to the negative resulting value when the input is $-2^{63}$ (REDFIN uses the common two's complement signed number representation). The overflow is flagged by setting 0verflow. We use SBV's symbolic if-then-else operation ite to merge two 
symbolic values - in this case two possible next states, one of which is a state with the Overflow flag set:

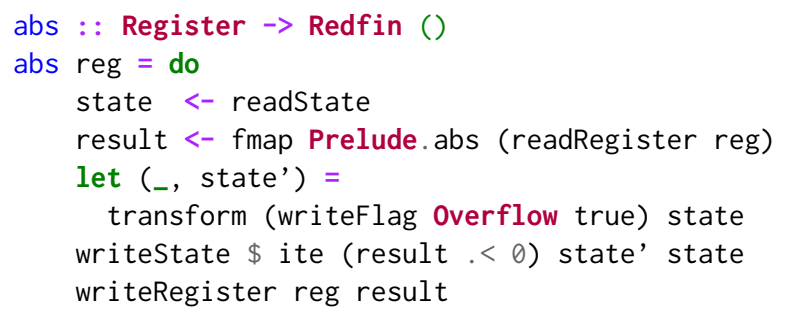

\subsubsection{Conditional Branching}

As an example of a control flow instruction consider jmpi_ct, which tests the Condition flag, and adds the provided off set to the instruction counter if the flag is set.

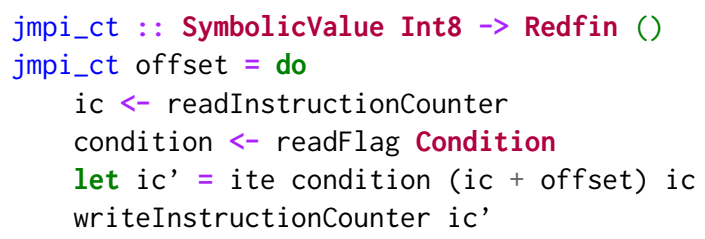

We use our Haskell encoding of the state transformer as a metalanguage: we operate the REDFIN core as a puppet master, using external meta-notions of addition, comparison and let-binding. From the processor's point of view, we have infinite memory and act instantly, which gives us unlimited modelling power. For example, we can simulate the processor environment in an external tool and feed its result to writeRegister as if it was obtained in one clock cycle.

\subsection{Symbolic Simulation}

Having defined the semantics of REDFIN programs, we can perform symbolic processor simulation. The function simulate takes a number of simulation steps $N$ and an initial symbolic State as input, and runs executeInstruction defined above $N$ times. In each state s we merge two possible futures: (i) if the Halt flag is set, we remain in the current state, since in this case the processor must remain idle; (ii) otherwise, we continue the simulation from the next state.

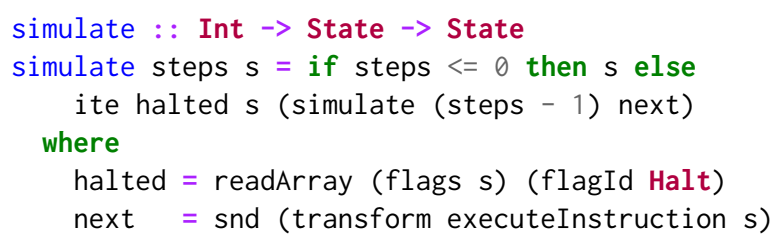

Symbolic simulation is very powerful. It allows us to formally verify properties of REDFIN programs by fixing some parts of the state to constant values (e.g., the program), and then making assertions on the resulting values of the symbolic part of the state, as demonstrated in the next section $\S 4$.

\section{Formal Verification}

This section presents a formal verification framework developed on top of the REDFIN semantic core (§3).
The verification workflow comprises the following steps:

- Develop programs in low-level REDFIN assembly, and in a high-level typed language embedded in Haskell.

- Test REDFIN programs on concrete input values.

- Define functional correctness and worst case execution time properties in the SBV property language.

- Verify the properties or obtain counterexamples.

Consider the following simple spacecraft control task.

Let $t_{1}$ and $t_{2}$ be two different time points (measured in $\mathrm{ms}$ ), and $p_{1}$ and $p_{2}$ be two power values (measured in $\mathrm{mW}$ ). Calculate the estimate of the total energy consumption during this period using linear approximation, rounding down to the nearest integer:

$$
\text { energyEstimate }\left(t_{1}, t_{2}, p_{1}, p_{2}\right)=\left\lfloor\frac{\left|t_{1}-t_{2}\right| *\left(p_{1}+p_{2}\right)}{2}\right\rfloor \text {. }
$$

This task looks too simple, but in fact it has a few pitfalls that, if left unattended, may lead to the failure of the space mission. Examples of subtle bugs in seemingly simple programs leading to a catastrophe include 64-bit to 16-bit number conversion overflow causing the destruction of Ariane 5 rocket [Ben-Ari 2001] and the loss of NASA's Mars orbiter due to incorrect unit conversion [NASA 1999]. Let us develop and verify a REDFIN program for this task.

We can write programs in the untyped REDFIN assembly, or in a typed higher-level expression language. The former allows engineers to hand-craft highly optimised programs under tight resource constraints, while the latter brings typesafety and faster prototyping. We start with the high-level approach and define an expression that can be used both as a Haskell function and a high-level REDFIN expression:

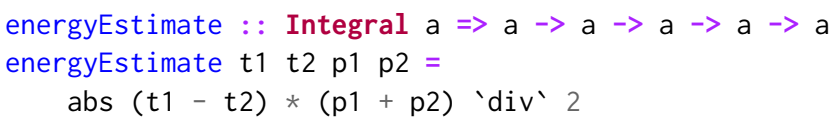

Thanks to polymorphism, we can treat energyEstimate both as a numeric function, and as an abstract syntax tree that can be compiled into a REDFIN assembly Script. Due to the lack of space we omit the implementation of Script, but one can think of it as a restricted version of the Redfin state transformer, which we use to write programs that can manipulate the processor state only by executing instructions, e.g. the only way to set the Overflow flag is to execute an arithmetic instruction that might cause an overflow.

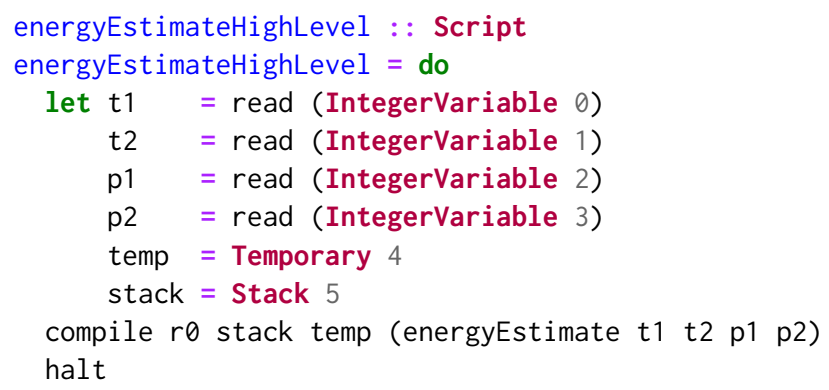


Here the type IntegerVariable is used to statically distinguish between integer and fixed-point numbers, Temporary to mark temporary words, so they cannot be mixed with inputs and outputs, and Stack to denote the location of the stack pointer. The let block declares six adjacent memory addresses: four input values $\left\{t_{1}, t_{2}, p_{1}, p_{2}\right\}$, a temporary word, and a stack pointer. We compile the high-level expression energyEstimate into the assembly language by translating it to a sequence of REDFIN instructions. The first argument of the compile function holds the register $r \theta$ which contains the estimated energy value after the program execution.

We can run symbolic simulation for 100 steps, initialising the program and data memory of the processor using the function simulate defined above and a helper function boot.

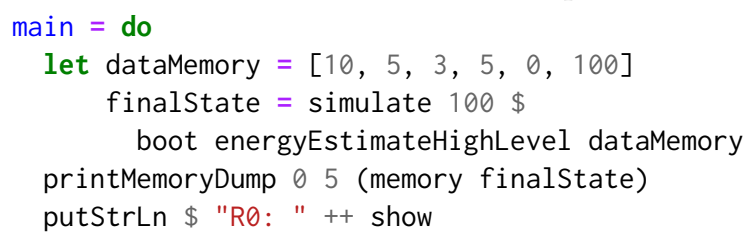

As the simulation result we get a finalstate. We inspect it by printing relevant components: the values of the first six memory cells, and the result of the computation located in the register $r 0$. Note that the stack pointer (cell 5) holds 100, as in the initial state, which means the stack is empty.

Memory dump: $[10,5,3,5,5,100]$

$\mathrm{R} 0: 20$

Simulating programs with specific inputs is useful for diagnostics and test, but SMT solvers allow us to verify the correctness for all valid input combinations. To demonstrate this, let us discover a problem in our energy estimation program. Consider the following correctness property.

Assuming that values $p_{1}$ and $p_{2}$ are non-negative integers, the energy estimation subroutine must always return a non-negative integer value.

To check that the program meets this requirement, we translate energyEstimateHighLevel into an SMT formula, and formulate the corresponding theorem:

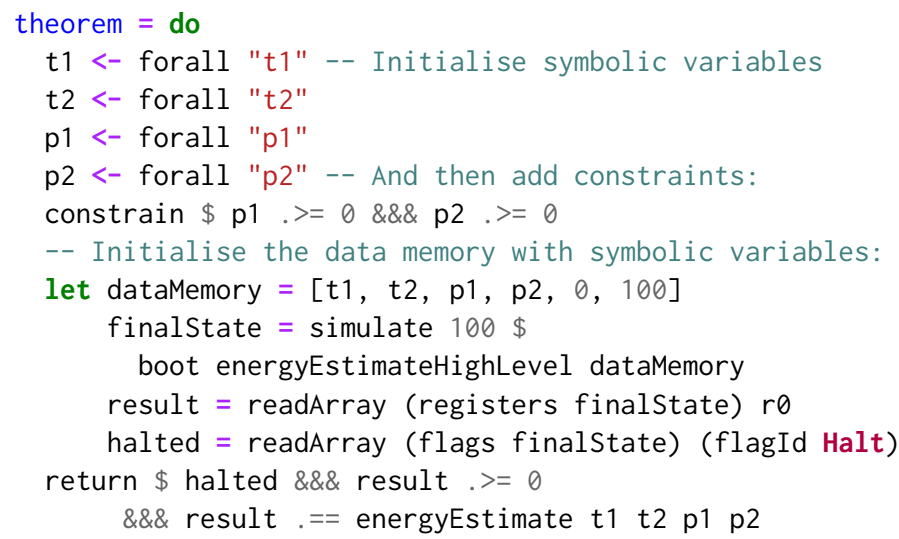

We extract the computed result and the value of the flag Halt from the finalstate, and then assert that the processor has halted, the result is non-negative, and is equal to that computed by the high-level Haskell expression energyEstimate. The resulting SMT formula can be checked by Z3 in $3.0 \mathrm{~s}^{2}$ :

\begin{tabular}{|l} 
> proveWith z3 theorem \\
Falsifiable. Counter-example: \\
t1 $=5190405167614263295::$ Int64 \\
t2 $=r:$ Int64 \\
p1 $=149927859193384455::$ Int64 \\
p2 $=157447350457463356::$ Int64
\end{tabular}

Z3 has found a counterexample demonstrating that the program does not satisfy the above property. Indeed, the expression evaluates to a negative value on the provided inputs due to an integer overflow. We therefore refine the property:

According to the spacecraft power system specification, $p_{1}$ and $p_{2}$ are non-negative integers not exceeding $1 \mathrm{~W}$. The time is measured from the mission start, hence $t_{1}$ and $t_{2}$ are non-negative and do not exceed the time span of the mission, which is 30 years. Under these assumptions, the energy estimation subroutine must return a non-negative integer value.

We need to modify time and power constraints accordingly:

$$
\begin{aligned}
& \text { constrain } \$ \mathrm{p} 1 .<=\text { toMilliWatts }(1:: \text { Watt }) \\
& \& \& \& \text { t } 1 .<=\text { toMilliSeconds }(30:: \text { Year }) \\
& \& \& \& \text { t } 1 .>=0 \& \& \& \text { t2 .>= } 0 \text { \&\&\& ... -- etc. }
\end{aligned}
$$

Rerunning Z3 produces the desired QED outcome in $4.8 \mathrm{~s}$.

The refinement has rendered the integer overflow impossible; in particular, abs can never be called with $-2^{63}$ within the mission parameters. Such guarantee fundamentally requires solving an SMT problem, even if it is done at the type level, e.g. using refinement types [Vazou et al. 2014].

The statically typed high-level expression language is very convenient for writing REDFIN programs, however, an experienced engineer can often find a way to improve the resulting code. In some resource-constrained situations, a fully hand-crafted assembly code may be required. As an example, consider the following low-level program:

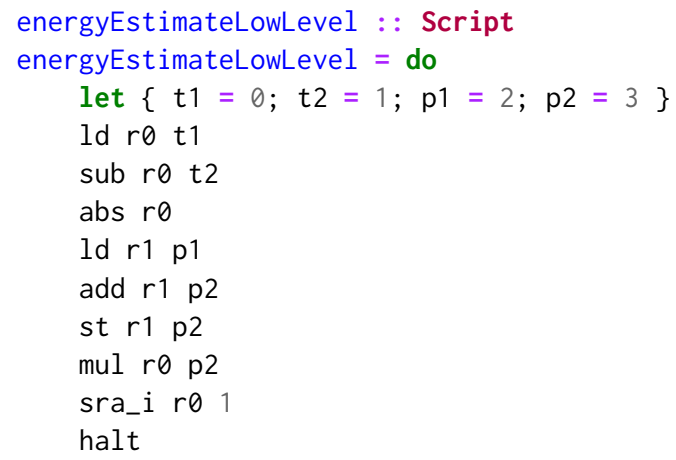

${ }^{2}$ We use a laptop with $2.90 \mathrm{GHz}$ Intel Core i5-4300U processor, 8GB RAM (3MB cache), and the SMT solver Z3 version 4.5.1 (64-bit). 
This program computes the energy estimate using only 9 instructions, whereas a direct unoptimised translation of the energyEstimate expression into assembly uses 79 instructions, most of them for stack manipulation.

To support the development of hand-crafted code, we use $\mathrm{Z} 3$ to check the equivalence of REDFIN programs by verifying that they produce the same output on all valid inputs. This allows an engineer to optimise a high-level prototype and have a guarantee that no bugs were introduced in the process.

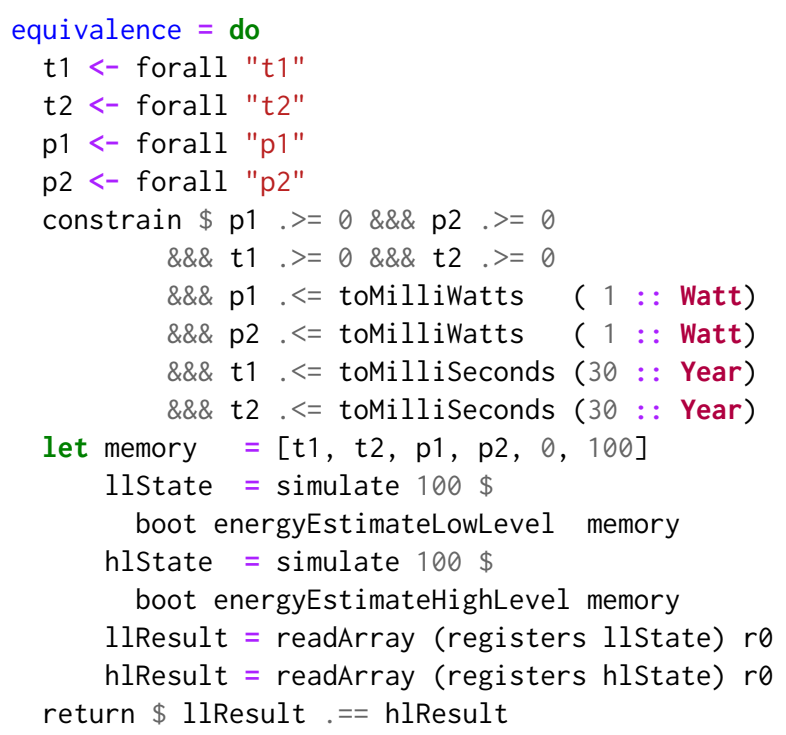

The equivalence check succeeds and takes $11.5 \mathrm{~s}$.

Every call of the executeInstruction function advances the clock field of the State (see Fig. 2) by the appropriate number of cycles, precisely matching the hardware implementation. This allows us to perform best/worst-case execution timing analysis using the optimisation facilities of SBV and Z3. As an example, let us determine the minimum and maximum number of clock cycles required for executing energyEstimateLowLevel. To make this example more interesting, we modified the semantics of the instruction abs and added 1 extra clock cycle in case of a negative argument.

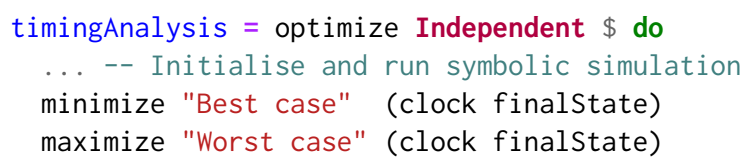

The total delay of the program depends only on the sign of $t_{1}-t_{2}$, thus the best and worst cases differ only by one clock cycle. The worst case is achieved when the difference is negative $\left(t_{1}-t_{2}=-2\right)$, as shown below. Z3 finishes in $0.5 \mathrm{~s}$.

\begin{tabular}{|c|c|c|c|}
\hline \multicolumn{2}{|c|}{$\begin{array}{l}\text { Objective "Best case": } \\
\text { Optimal model: }\end{array}$} & \multicolumn{2}{|c|}{$\begin{array}{l}\text { Objective "Worst case": } \\
\text { Optimal model: }\end{array}$} \\
\hline t1 & $=549755813888$ & t1 & 65535 \\
\hline t2 & $=17179869184$ & t2 & 65537 \\
\hline $\mathrm{p} 1$ & $=0$ & $\mathrm{p} 1$ & 0 \\
\hline p2 & 0 & p2 & 0 \\
\hline Best case $=$ & 12 & Worst case $=$ & 13 \\
\hline
\end{tabular}

\section{Discussion}

As the previous section $\S 4$ demonstrates, the presented approach provides a unified specification, testing, and formal verification framework. It allows the REDFIN engineering team to co-develop REDFIN software and hardware, by extending and modifying the default instruction semantics. By using Haskell as a metalanguage, one can implement higherlevel languages on top of the REDFIN assembly, such as our simple statically-typed language for arithmetic expressions.

Static typing, polymorphism, do-notation, and availability of a mature symbolic manipulation library (SBV) were the key factors for choosing Haskell for this project. We also have a prototype implementation in the dependently-typed language Idris [Brady 2013] that allows us to verify more sophisticated properties at the type level, however at the time of writing there is no equivalent of the SBV library in Idris, which is a significant practical disadvantage.

The Script monad was engineered to provide familiar assembly mnemonics and directives (e.g. labels), which allows engineers to start using the framework for developing REDFIN programs even without prior Haskell experience, hopefully increasing the uptake of the framework.

Thanks to symbolic simulation, we can uniformly handle both concrete and symbolic values, reusing the same code base and infrastructure for testing and formal verification. Testing yields trivial SMT problems that can be solved in subsecond time. Formal verification is more expensive: in our experiments, realistic programs (e.g. for controlling a stepper motor in antenna and solar panel positioning units, with a loop and $\approx 100$ instructions) required 10 -30 minutes, but one can easily construct tiny programs that will grind any SMT solver to a halt: for example, analysis of a single multiplication instruction can take half an hour if it is required to factor 64-bit numbers - try to factor 4611686585363088391 with an SMT solver! In such cases, conservatively proving some of the correctness properties at the type level can significantly increase the productivity. As a microbenchmark, we verified the correctness of an array summation program, reporting the number of SMT clauses and Z3 runtime for low-level (LL) and high-level (HL) programs:

\begin{tabular}{l||c|c|c|c|c}
\hline Benchmark & $\begin{array}{c}\text { Array } \\
\text { size }\end{array}$ & $\begin{array}{c}\text { Clauses } \\
\text { LL }\end{array}$ & $\begin{array}{c}\text { Clauses } \\
\text { HL }\end{array}$ & $\begin{array}{c}\text { Time } \\
\text { LL }\end{array}$ & $\begin{array}{c}\text { Time } \\
\text { HL }\end{array}$ \\
\hline Overflow: & 9 & 286 & 260 & $1.482 \mathrm{~s}$ & $0.443 \mathrm{~s}$ \\
values not & 12 & 492 & 453 & $3.604 \mathrm{~s}$ & $1.365 \mathrm{~s}$ \\
constrained & 15 & 740 & 688 & $49.969 \mathrm{~s}$ & $7.362 \mathrm{~s}$ \\
& 18 & 1030 & 965 & $76.757 \mathrm{~s}$ & $88.458 \mathrm{~s}$ \\
\hline No overflow: & 9 & 318 & 292 & $0.467 \mathrm{~s}$ & $0.119 \mathrm{~s}$ \\
all values & 12 & 549 & 510 & $0.739 \mathrm{~s}$ & $0.682 \mathrm{~s}$ \\
in [1,1000] & 15 & 828 & 776 & $1.839 \mathrm{~s}$ & $6.408 \mathrm{~s}$ \\
& 18 & 1155 & 1090 & $9.944 \mathrm{~s}$ & $72.880 \mathrm{~s}$ \\
\hline Equivalence & 9 & 258 & 261 & $0.039 \mathrm{~s}$ & $0.097 \mathrm{~s}$ \\
to the sum & 12 & 495 & 459 & $0.053 \mathrm{~s}$ & $0.647 \mathrm{~s}$ \\
function & 15 & 708 & 702 & $0.311 \mathrm{~s}$ & $7.322 \mathrm{~s}$ \\
& 18 & 1005 & 990 & $2.633 \mathrm{~s}$ & $71.896 \mathrm{~s}$ \\
\hline
\end{tabular}




\section{Related Work}

There is a vast body of literature available on the topic of formal verification, including verification of hardware processing cores and low-level software programs. Our work builds in a substantial way on a few known ideas that we will review in this section. We thank the formal verification and programming languages communities and hope that the formal semantics of the REDFIN processing core will provide a new interesting benchmark for future studies.

We model the REDFIN microarchitecture using a monadic state transformer metalanguage - an idea with a long history. Fox and Myreen [2010] formalise the Arm v7 instruction set architecture in HOL4 and give a careful account to bit-accurate proofs of the instruction decoder correctness. Later, Kennedy et al. [2013] formalised a subset of the x 86 architecture in Coq, using monads for instruction execution semantics, and do-notation for assembly language embedding. Degenbaev [2012] formally specified the complete $\mathrm{x} 86$ instruction set - a truly monumental effort! - using a custom domain-specific language that can be translated to a formal proof system. Arm's Architecture Specification Language (ASL) has been developed for the same purpose to formalise the Arm v8 instruction set [Reid et al. 2016]. The SAIL language [Armstrong et al. 2019] has been designed as a generic language for ISA specification and was used to specify the semantics of ARMv8-A, RISC-V, and CHERI-MIPS. Our specification approach is similar to these three works, but we operate on a much smaller scale of the REDFIN core and focus on verifying whole programs.

Our metalanguage is embedded in Haskell and does not have a rigorous formalisation, i.e. we cannot prove the correctness of the REDFIN semantics itself, which is a common concern, e.g. see Reid [2017]. Moreover, our verification workflow mainly relies on automated theorem proving, rather than on interactive one. This is motivated by the cost of precise proof assistant formalisations in terms of human resources: automated techniques are more CPU-intensive, but cause less "human-scaling issues" [Reid et al. 2016]. Our goal was to create a framework that could be seamlessly integrated into an existing spacecraft engineering workflow, therefore it needed to have as much proof automation as possible. The automation is achieved by means of symbolic program execution. Currie et al. [2006] applied symbolic execution with uninterpreted functions to prove equivalence of low-level assembly programs. The framework we present allows not only proving the equivalence of low-level programs, but also their compliance with higher-level specifications written in a subset of Haskell.

Finally, we would like to acknowledge the projects and talks that provided an initial inspiration for this work: the 'Monads to Machine Code' compiler by Diehl [2017], RISC-V semantics by MIT [2017], the assembly monad by Wall [2017], and SMT-based program analysis by Jelvis [2016].

\section{Acknowledgements}

We would like to thank Vitaly Bragilevsky, Neil Mitchell, Charles Morisset, Artem Pelenitsyn, Danil Sokolov, as well as the three Haskell Symposium reviewers for their helpful feedback on an earlier version of this paper.

\section{References}

Alasdair Armstrong, Thomas Bauereiss, Brian Campbell, Alastair Reid, Kathryn E. Gray, Robert M. Norton, Prashanth Mundkur, Mark Wassell, Jon French, Christopher Pulte, Shaked Flur, Ian Stark, Neel Krishnaswami, and Peter Sewell. 2019. ISA Semantics for ARMv8-a, RISC-v, and CHERIMIPS. Proc. ACM Program. Lang. 3, POPL, Article 71 (Jan. 2019), 31 pages. https://doi.org/10.1145/3290384

Mordechai Ben-Ari. 2001. The Bug That Destroyed a Rocket. SIGCSE Bull. 33, 2 (June 2001), 58-59.

Edwin Brady. 2013. Idris, a general-purpose dependently typed programming language: Design and implementation. Fournal of Functional Programming 23 (9 2013), 552-593. Issue 05.

David Currie, Xiushan Feng, Masahiro Fujita, Alan J. Hu, Mark Kwan, and Sreeranga Rajan. 2006. Embedded Software Verification Using Symbolic Execution and Uninterpreted Functions. International fournal of Parallel Programming 34, 1 (2006), 61-91.

Leonardo De Moura and Nikolaj Bjørner. 2008. Z3: An efficient SMT solver. Tools and Algorithms for the Construction and Analysis of Systems (2008), 337-340.

Ulan Degenbaev. 2012. Formal specification of the $x 86$ instruction set architecture. Ph.D. Dissertation. Saarland University.

Stephen Diehl. 2017. Monads to Machine Code. https://web.archive.org/ web/20171207020256/http://www.stephendiehl.com/posts/monads_ machine_code.html.

Levent Erkok. 2019. SBV: SMT Based Verification in Haskell. http:// leventerkok.github.io/sbv/

Anthony Fox and Magnus O Myreen. 2010. A trustworthy monadic formalization of the ARMv7 instruction set architecture. In International Conference on Interactive Theorem Proving. Springer, 243-258.

Tikhon Jelvis. 2016. Analyzing Programs with Z3 (video recording of Compose Conference talk). https://web.archive.org/web/20170205204536/http: //jelv.is/talks/compose-2016/.

Andrew Kennedy, Nick Benton, Jonas B Jensen, and Pierre-Evariste Dagand. 2013. Coq: the world's best macro assembler?. In Proceedings of the 15th Symposium on Principles and Practice of Declarative Programming. ACM, 13-24.

Nancy G. Leveson. 2004. Role of Software in Spacecraft Accidents. fournal of Spacecraft and Rockets 41, 4 (2004), 564-575.

MIT. 2017. A formal specification of the RISC-V ISA written in Haskell. https://github.com/mit-plv/riscv-semantics.

NASA. 1999. Mars Climate Orbiter Mishap Investigation Board Phase I Report. Technical Report.

Alastair Reid. 2017. Who Guards the Guards? Formal Validation of the Arm V8-m Architecture Specification. Proc. ACM Program. Lang. 1, OOPSLA (2017), 88:1-88:24.

Alastair Reid, Rick Chen, Anastasios Deligiannis, David Gilday, David Hoyes, Will Keen, Ashan Pathirane, Owen Shepherd, Peter Vrabel, and Ali Zaidi. 2016. End-to-end verification of processors with ISA-Formal. In International Conference on Computer Aided Verification. Springer, 42-58.

Niki Vazou, Eric L Seidel, Ranjit Jhala, Dimitrios Vytiniotis, and Simon Peyton-Jones. 2014. Refinement types for Haskell. In ACM SIGPLAN Notices, Vol. 49. ACM, 269-282.

Philip Wadler. 1990. Comprehending monads. In Proceedings of the 1990 ACM conference on LISP and functional programming. ACM, 61-78.

Lewis Wall. 2017. An ASM Monad. https://web.archive.org/web/ 20190519181416/http://wall.org/ lewis/2013/10/15/asm-monad.html. 